\title{
Restoration of Regulatory T-Cell Function in Dry Eye Disease by Antagonizing Substance P/Neurokinin-1 Receptor
}

Yukako Taketani, ${ }^{*}$ Anna Marmalidou, ${ }^{* \dagger}$ Thomas H. Dohlman, ${ }^{*}$ Rohan Bir Singh, ${ }^{*}$ Afsaneh Amouzegar, ${ }^{*}$ Sunil K. Chauhan, ${ }^{*}$ Yihe Chen, * and Reza Dana*

From the Schepens Eye Research Institute of Massachusetts Eye and Ear* and the Department of Ophthalmology, ${ }^{\dagger}$ Massachusetts Eye and Ear, Harvard Medical School, Boston, Massachusetts; and the Department of Internal Medicine, ${ }^{\ddagger}$ University of Pittsburgh School of Medicine, Pittsburgh, Pennsylvania

Accepted for publication

May 5, 2020.

Address correspondence to Reza Dana, M.D., M.Sc., M.P.H., or Yihe Chen, M.D., Schepens Eye Research Institute, Massachusetts Eye and Ear, Department of Ophthalmology, Harvard Medical School, 20 Staniford St., Boston, MA 02114. E-mail: reza dana@meei.harvard.edu or yihe_chen@meei.harvard.edu.

\begin{abstract}
Substance $\mathrm{P}(\mathrm{SP})$ is a tachykinin neuropeptide, implicated in the pathogenesis of various inflammatory conditions and a critical mediator in pain transmission. Recently, the role of SP was described in the pathogenesis of dry eye disease (DED) through its role in the maturation of antigen-presenting cells at the ocular surface after exposure to desiccating stress. However, the effect of SP on regulatory T cells (Tregs), which are functionally impaired in DED, remains unclear. This study examined the phenotypic and functional changes in Tregs in response to SP in DED. The in vitro cultures of normal Tregs in the presence of SP led to a significant reduction in both Treg frequencies and their suppressive function, which was prevented by the addition of an SP receptor (neurokinin-1 receptor) antagonist. Furthermore, in vivo treatment with the neurokinin-1 receptor antagonist in DED mice effectively restored Treg function, suppressed pathogenic T helper 17 response, and significantly ameliorated the disease. Our results show that a significant increase in SP levels promotes Treg dysfunction in DED, and blockade of SP effectively restores Treg function and suppresses DED severity. (Am J Pathol 2020, 190: 1859-1866; https://doi.org/10.1016/j.ajpath.2020.05.011)
\end{abstract}

Dry eye disease (DED) is a highly prevalent multifactorial ocular surface disorder that presents either as a stand-alone disease or as an ocular manifestation of a systemic disease such as graft-versus-host disease, Sjögren syndrome, and rheumatoid arthritis. ${ }^{1-5}$ Studies have calculated the prevalence of symptomatic DED in the United States to be $14.5 \%$, and the total annual cost for the management of this disease is estimated to be near $\$ 4$ billion. ${ }^{6,7}$

There is ample evidence indicating the role of autoimmune inflammation in the pathogenesis of DED. ${ }^{8}$ Multiple studies, $^{9-12}$ have shown that inflammation in DED is principally mediated by the unrestrained effector $\mathrm{T}$ helper 17 (Th17) response resulting from dysfunction of the immunosuppressive regulatory $\mathrm{T}$ cells (Tregs).

Substance P (SP, also known as TAC1) is an 11 amino acid neuropeptide belonging to the tachykinin family. ${ }^{13}$ It is known to mediate neurotransmission of pain, as well as neuro-inflammation, by binding primarily to the neurokinin
1 receptor (NK-1R). ${ }^{14,15}$ It is secreted by sensory nerve endings, including corneal trigeminal nerves, and functions by binding to NK-1R. ${ }^{16-19} \mathrm{SP}$ is also secreted by corneal epithelial cells and various immune cells, including macrophages, monocytes, and dendritic cells during inflammation. ${ }^{20-25}$ SP has known pro-inflammatory effects and plays a role in the pathogenesis of autoimmune disorders such as rheumatoid arthritis and inflammatory bowel disease, where dysfunction of Tregs is similarly involved in disease pathogenesis as in DED. ${ }^{12,26-28}$ Recent studies showed the role played by increased levels of SP on the ocular surface in promoting the maturation of corneal antigen-presenting

Supported by the National Eye Institute/NIH grant R01 EY020889 (R.D.), and National Eye Institute/NIH core grant P30EY003790.

Disclosures: R.D. and Y.C. are named as inventors in a patent filing (US Patent Application No. 62/879,839) owned by Massachusetts Eye and Ear related to targeting substance $\mathrm{P}$ in ocular surface diseases. 
cells, which subsequently migrate to the draining lymph nodes (DLNs) and activate effector Th17 cells in DED. ${ }^{29}$ However, the precise function of SP on Tregs in autoimmunity or other inflammatory conditions remains unknown.

In the current study, we first evaluated the expression levels of SP in the DLNs and NK-1R by Tregs in DED mice. The results show that SP levels as well as the frequencies of NK-1 $\mathrm{R}^{+}$Tregs in DLNs are increased in DED. To test the hypothesis that SP directly influences Tregs through NK-1R, we conducted in vitro experiments of Tregs in the presence of SP. We also investigated the effects of inhibiting SP signaling by using NK-1R antagonists on Treg function and on recovery of regulatory function.

\section{Materials and Methods}

\section{Animals}

Eight-to-ten-week-old C57BL/6 female mice (Charles River Laboratories, Wilmington, MA) were used in all of the experiments. The protocol was approved by the Schepens Eye Research Institute Animal Care and Use Committee. All animals were treated in accordance with the Association for Research in Vision and Ophthalmology Statement for the use of animals in ophthalmic and vision research. ${ }^{30}$

\section{Induction of DED and Clinical Evaluation}

DED was induced by continuously exposing the mice to desiccating stress $(<20 \%$ relative humidity with an airflow of $15 \mathrm{~L} / \mathrm{min}$ at a temperature of $21^{\circ} \mathrm{C}$ to $23^{\circ} \mathrm{C}$ ) in a controlled-environment chamber for 14 days. ${ }^{31}$ Age- and sex-matched mice (8-to-10-week-old; C57BL/6 female) placed in the standard vivarium were used as control mice. Corneal fluorescein staining (CFS) was used to evaluate corneal epitheliopathy. Briefly, $1 \mu \mathrm{L}$ of $2 \%$ fluorescein was applied to the lateral conjunctival sac of the mice, and slit lamp examination was performed under cobalt blue light. Punctate staining was recorded in a masked fashion by using the standard National Eye Institute grading system with the score ranging from 0 to 3 for each of the five areas of the cornea. $^{32}$

\section{Isolation of Tregs and in Vitro Culture}

The submandibular and cervical DLNs were harvested from normal and DED mice, and single-cell suspensions were prepared as previously described. ${ }^{12} \mathrm{CD} 4{ }^{+} \mathrm{CD} 25^{+}$Tregs were isolated by magnetic separation using a Treg isolation kit (Miltenyi Biotec, Somerville, MA). The purity of sorted cells $\left(>95 \%\right.$ Foxp $^{+}$Tregs) was confirmed by using flow cytometry analysis. Tregs were resuspended in RPMI 1640-based complete medium at a concentration of $1 \times 10^{6}$ cells $/ \mathrm{mL}$. Subsequently, $100 \mu \mathrm{L}$ of cell suspension was plated in each well of a 96-well plate (Corning Life Sciences Inc., Tewksbury, MA). SP $(1 \mu \mathrm{mol} / \mathrm{L}$; Tocris Bioscience, Minneapolis, MN), Spantide I $(10 \mu \mathrm{mol} / \mathrm{L}$; Tocris Bioscience), or both were added to the Treg cultures. After 48 hours of incubation, cells were collected for flow cytometry analysis.

\section{Flow Cytometry Analysis}

Single-cell suspensions were prepared from conjunctival tissues of mice, as previously described. ${ }^{33}$ Briefly, conjunctivae were harvested and cut into small fragments, followed by digestion with $2 \mathrm{mg} / \mathrm{mL}$ collagenase type IV (MilliporeSigma, Burlington, MA) and $0.05 \mathrm{mg} / \mathrm{mL}$ DNase I (Roche Holding AG, Basel, Switzerland) for 1 hour at $37^{\circ} \mathrm{C}$ with agitation. The suspension was then filtered through a $70-\mu \mathrm{m}$ cell strainer (BD Biosciences Inc., San Jose, CA). Single-cell suspensions from DLNs were prepared by homogenizing tissues and subsequently filtering them through a $70-\mu \mathrm{m}$ cell strainer. Cells were stained with fluorescein isothiocyanate-conjugated anti-CD4, phycoerythrin (PE)/Cy7-conjugated Foxp3, APCconjugated cytotoxic $\mathrm{T}$ lymphocyte associated antigen-4 (CTLA-4) or lymphocyte activation gene-3, and PEconjugated programmed cell death 1 (PD-1) or glucocorticoid-induced tumor necrosis factor receptor (GITR) (BioLegend Inc., San Diego, CA) for flow cytometry analysis. NK-1R was stained with primary antibody (Santa Cruz Biotechnology Inc., Dallas, TX) and secondary antibody (fluorescein isothiocyanate; Jackson ImmunoResearch Laboratories, Inc., West Grove, PA). For intracellular IL-17A staining, the cells were stimulated with $50 \mathrm{ng} / \mathrm{mL}$ phorbol myristate acetate and $500 \mathrm{ng} / \mathrm{mL}$ ionomycin (MilliporeSigma) for 6 hours at $37^{\circ} \mathrm{C}$ and $5 \%$ carbon dioxide in the presence of GolgiStop (BD Biosciences Inc.) to inhibit cytokine secretion before staining and then stained with PE-Cy7-conjugated anti-IL-17A antibody (BioLegend Inc.). The stained cells were examined with an LSR II flow cytometer (BD Biosciences Inc.) and the results analyzed. Data were analyzed by using FlowJo software version X.5.3 (FlowJo LLC., Ashland, OR).

\section{Treg Suppression Assay}

The suppressive function of Tregs was examined by using the suppression assay, as previously described. ${ }^{12}$ Briefly, Tregs and responder $\mathrm{CD}^{+}{ }^{+} \mathrm{T}$ (Tresp) cells were isolated from the DLNs. Purified total CD4 ${ }^{+}$Tresp cells $\left(1 \times 10^{5}\right)$ were co-cultured with $\mathrm{CD} 4{ }^{+} \mathrm{CD} 25^{+}$Tregs $\left(5 \times 10^{4}\right)$, T cell-depleted syngeneic splenocytes $\left(1 \times 10^{5}\right)$, and $1 \mu \mathrm{g} /$ $\mathrm{mL}$ anti-CD3 antibody for 3 days. Proliferation of Tresp cells without adding Tregs was considered as control with $0 \%$ suppression. The proliferation was measured by using the BrdU proliferation kit (MilliporeSigma), and the percentage of suppression was calculated by using the following formula: 


$$
\% \text { suppression }=\frac{(\text { Tresp proliferation without Tregs }- \text { Tresp proliferation with Tregs })}{\text { Tresp proliferation without Tregs }} \times 100
$$

\section{Real-Time PCR}

DLNs were harvested from mice, and total RNA was isolated by using the RNeasy Micro kit (Qiagen Inc., Germantown, MD) as per the manufacturer's recommendations and reverse transcribed by using an RT transcription kit (Qiagen Inc.). Real-time PCR was performed by using the TaqMan Universal PCR Master mix and predesigned primers SP (Tac1, Mm01166996_m1) and Gapdh (Mm99999915_g1) (Thermo Fisher Scientific, Waltham, MA) in a Realplex2 system (Eppendorf GmbH, Hamburg, Germany). The Gapdh gene was used as an endogenous control for each reaction. The results of quantitative PCR were analyzed by using the comparative CT method in which the target change $=\Delta \Delta \mathrm{CT}$. The results were normalized by the $\mathrm{CT}$ value of Gapdh, and the mean CT of the relative mRNA level in the control group was used as the calibrator.

\section{Cytokine Expression Assay}

For protein extraction, DLNs were harvested and homogenized in radioimmunoprecipitation assay buffer containing protease inhibitors (MilliporeSigma) on ice and centrifuged. The supernatant was assayed by using commercial enzyme-linked immunosorbent assay kits for SP (detection limit, 43.8 pg/mL; R\&D Systems, Minneapolis, $\mathrm{MN}$ ), IL-10, and transforming growth factor- $\beta$ (TGF- $\beta$ ) (BioLegend Inc.), as well as total protein amount (Thermo Fisher Scientific).

\section{In Vivo Treatment of Mice with Spantide I}

DED mice were injected intraperitoneally with $36 \mu \mathrm{g}$ synthetic Spantide I or vehicle control [phosphate-buffered saline (PBS)] once daily in $100 \mu \mathrm{L}$ volume starting from one day before DED induction (ie, day -1), until day 14. CFS scoring was recorded on days $0,3,7,10$, and 14 to assess disease severity. On day 14 , the mice were euthanized, and the tissues were harvested for further flow cytometry and enzyme-linked immunosorbent assay analyses.

\section{Statistical Analysis}

The $t$-test or $U$-test was used for comparison of means between the groups, and $P<0.05$ was considered statistically significant. The data are presented as mean \pm SEM of at least three independent experiments. Prism for Windows software version 8.0 (GraphPad Software, La Jolla, CA) was used for statistical analysis.

\section{Results}

\section{SP Expression Levels Increase in DLNs in DED}

DLNs are the principal site where Tregs normally regulate effector T cells; however, Tregs have been shown to become dysfunctional in DED. ${ }^{12}$ Thus, the association between Treg dysfunction and altered SP levels in the draining lymphoid tissues in DED was first investigated. After 14 days of DED induction, the mRNA expression levels of SP (Tacl) in DLNs increased more than fourfold (Figure 1A), associated with a $25 \%$ increase in SP protein levels (Figure 1B). There was no significant change in SP levels on day 7 after DED induction.
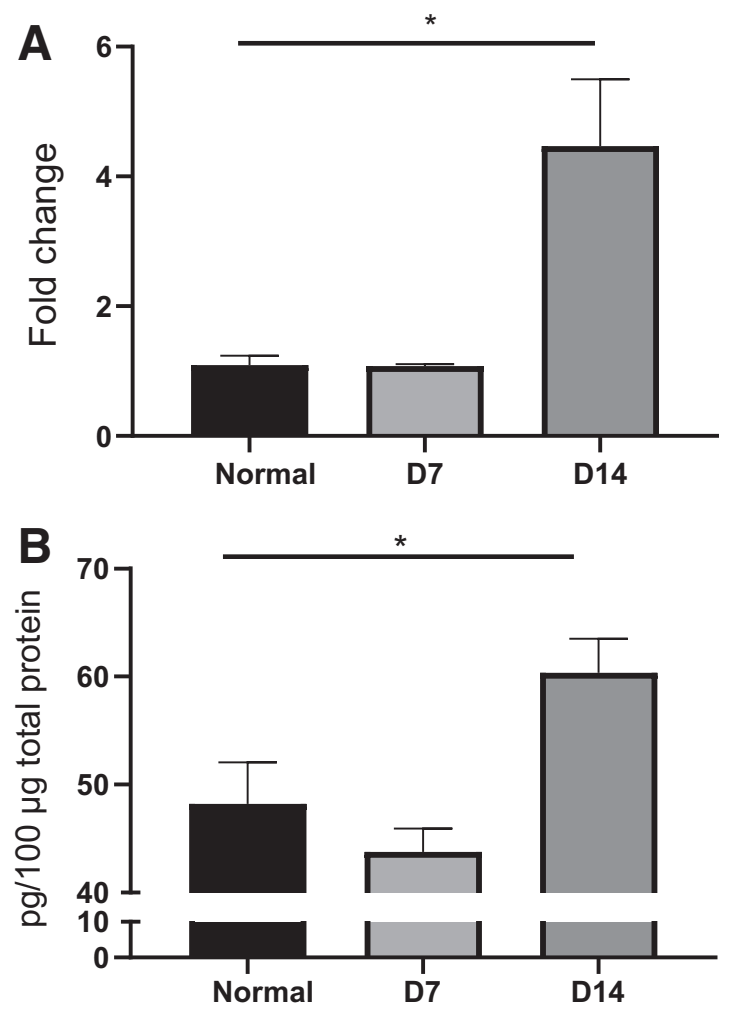

Figure 1 Expression of substance $P$ (SP, also known as Tac1) at draining lymph nodes in dry eye disease (DED). The expression levels of SP in draining lymph nodes of normal and DED mice (after 7 and 14 days of induction) were quantified by real-time quantitative PCR data for mRNA [the $y$ axis is fold change of SP expression compared with the normal group (normalized as 1.0)] (A) and by enzyme-linked immunosorbent assay for protein level (the $y$ axis indicates the concentration of SP in lymph nodes per $100 \mu \mathrm{g}$ of protein) (B). Both mRNA and protein level in DED mice at day 7 (D7) are the same as the normal group but increase significantly in DED mice at day 14 (D14) $(P=0.04$ and 0.049 , respectively). Data are expressed as means \pm SEM of three independent experiments. $n=5$ mice per group (A and $\mathbf{B}) .{ }^{*} P<0.05$. 


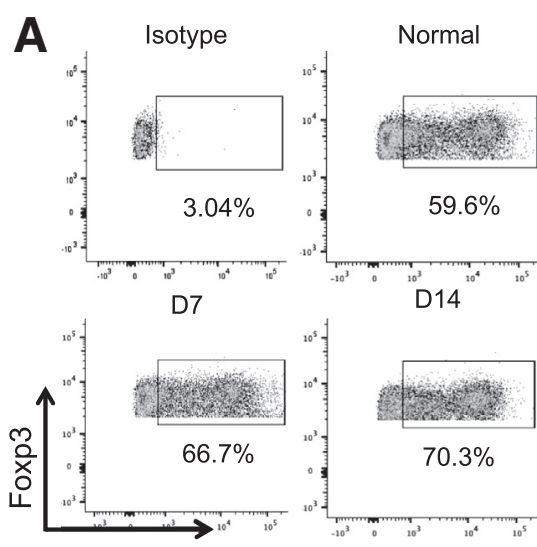

NK-1R

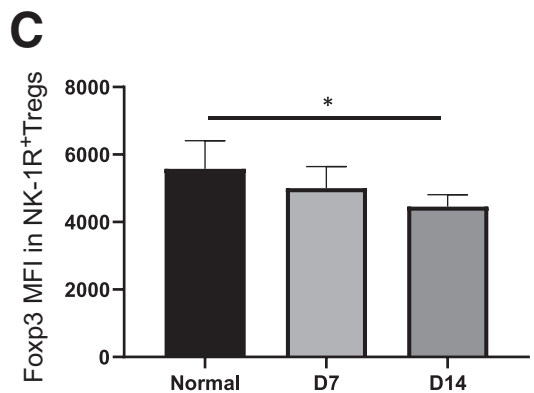

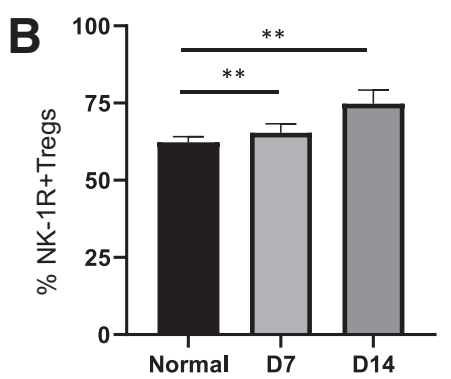

D

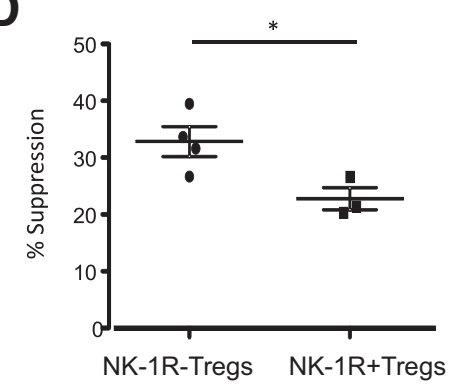

Figure 2 Neurokinin-1 receptor (NK-1R)-positive regulatory $T$ cells (Tregs) increase in the course of dry eye disease (DED) and exhibit impaired suppressive function. A and B: Representative flow cytometry plots (A) and summary data (B) showing frequencies of NK-1 ${ }^{+}$Tregs $\left(\mathrm{CD}^{+}{ }^{+} \mathrm{CD}_{25} 5^{+} \mathrm{Foxp}^{+}\right)$in draining lymph nodes of normal and DED mice after 7 and 14 days of DED induction. C: Expression levels of Foxp3 by NK-1 $\mathrm{R}^{+}$ Tregs in different groups are assessed by using mean fluorescence intensity (MFI). D: Treg function is assessed by using the suppression assay. Data are expressed as means \pm SEM of three independent experiments. $n=5$ mice per group $(\mathbf{A}-\mathbf{C}): n=7$ mice per group (D). ${ }^{*} P<0.05,{ }^{*} P<0.01$. D7, day 7; D14, day 14; Foxp3, forkhead box P3.
Frequency of Functionally Impaired, SP-Binding NK$1 \mathrm{R}^{+}$Treg Increases in DED

After detecting increased levels of SP in DLNs in DED, the frequencies of Tregs expressing the SP receptor (NK-1R) in DED were evaluated. As shown in Figure 2, A and B, the frequencies of $\mathrm{NK}-1 \mathrm{R}^{+}$Tregs progressively increased in DED, with $65.4 \%$ NK-1 $\mathrm{R}^{+}$Tregs on day 7 and $74.8 \%$ NK$1 \mathrm{R}^{+}$Tregs on day 14 , compared with $50.4 \%$ in normal control. Because Foxp3 is the critical transcriptional factor determining the immunosuppressive functions of Tregs, its expression levels on $\mathrm{NK}-1 \mathrm{R}^{+}$Tregs were examined. ${ }^{34,35}$
The results showed that Foxp3 expression levels were significantly decreased in NK-1 $\mathrm{R}^{+}$Tregs derived from DED mice (Figure 2C). Furthermore, Treg suppression assay showed that the capacity to suppress effector T-cell proliferation was significantly reduced in $\mathrm{NK}-1 \mathrm{R}^{+}$Tregs compared with NK-1 $\mathrm{R}^{-}$Tregs (Figure 2D).

\section{SP Induces Treg Dysfunction in Vitro and Is Reversed by the NK-1R Antagonist Spantide I}

After establishing elevated levels of SP expression and increased frequencies of NK-1 $\mathrm{R}^{+}$Tregs in DED, the direct
A
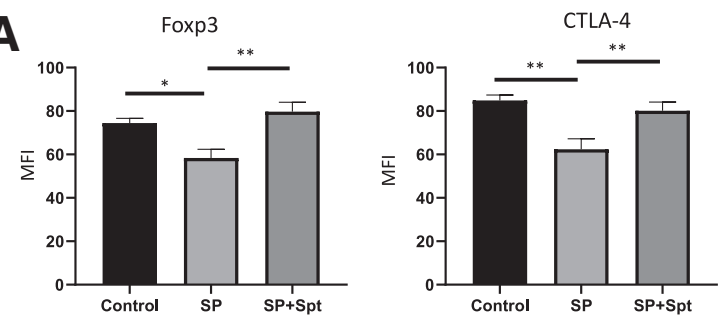

B

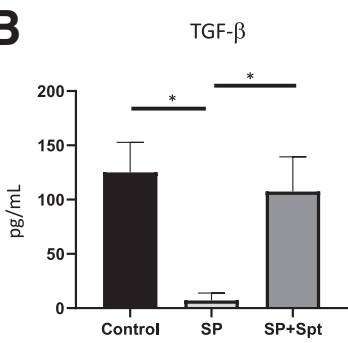

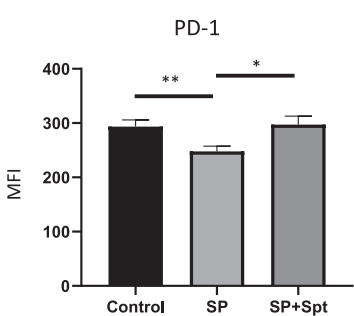

C

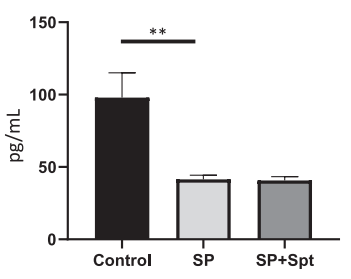

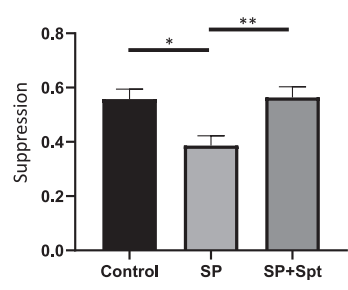

Figure 3 Substance $P$ directly impairs regulatory $T$ cell (Treg) function in vitro. Tregs were isolated from lymph nodes of normal mice and incubated in the presence of Substance $P(S P ; 1$ $\mu \mathrm{mol} / \mathrm{L}$ ), with or without Spantide I (Spt; 10 $\mu \mathrm{mol} / \mathrm{L}$ ) for 48 hours. Phosphate-buffered saline was used as a control. A: Flow cytometry analysis of cultured Tregs showing mean fluorescence intensities (MFIs) of forkhead box P3 (Foxp3), cytotoxic $T$ lymphocyte associated antigen-4 (CTLA-4), and programmed cell death 1 (PD-1) expression by Tregs. B: Protein levels of transforming growth factor- $\beta$ (TGF- $\beta$ ) and IL-10 in culture supernatants are quantified by using enzyme-linked immunosorbent assay. C: Suppressive functions of cultured Tregs are assessed by using Treg suppression assay. Data are expressed as means \pm SEM of three independent experiments., $n=5$ mice per group (A); $n=4$ mice per group (B); $n=9$ mice per group (C). ${ }^{*} P<0.05$, $* * P<0.01$. 

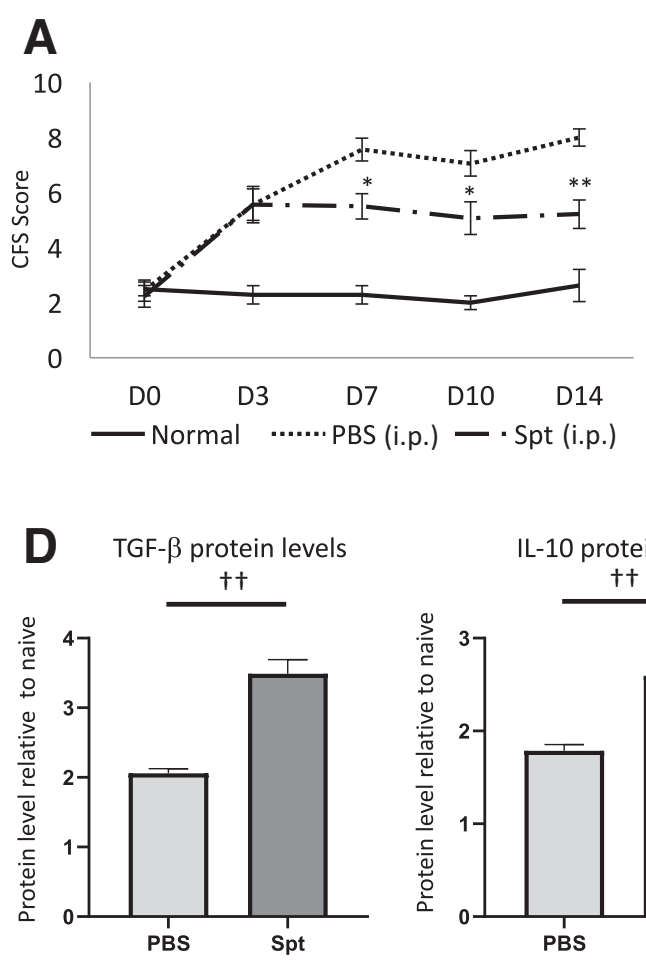
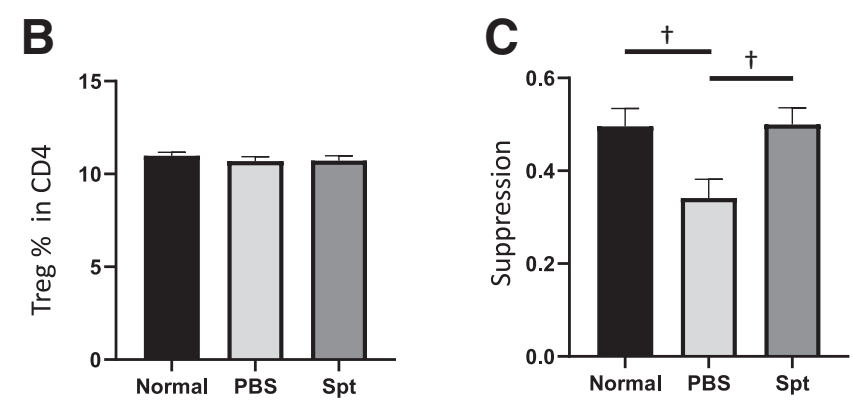

Figure 4 In vivo blockade of substance $\mathrm{P}$ (SP) signaling through systemic administration of Spantide I (Spt) preserves regulatory T cell (Treg) function and reduces severity of dry eye disease (DED). DED mice were injected intraperitoneally (i.p.) with $36 \mu \mathrm{g}$ of synthetic Spt or phosphate-buffered saline (PBS) once daily in 100 $\mu \mathrm{L}$ volume starting from one day before DED induction (day -1) until day 14. A: Corneal epitheliopathy in DED is assessed by standard corneal fluorescein staining (CFS) scores. B: Frequencies of $\mathrm{CD}^{+}{ }^{+} \mathrm{CD} 25^{+} \mathrm{Foxp} 3^{+}$Tregs as a proportion of total $\mathrm{CD}^{+}{ }^{+} \mathrm{T}$ cells in draining lymph nodes are analyzed by using flow cytometry at day 14. C: Suppressive functions of Tregs are assessed. D: Fold increase in the protein levels of transforming growth factor- $\beta$ (TGF- $\beta$ ) and IL-10 in draining lymph nodes as evaluated by enzyme-linked immunosorbent assay. Data shown are the relative change to normal animals. Data are expressed as means \pm SEM of three independent experiments. $n=7$ mice per group (A); $n=5$ mice per group (B and C); $n=4$ mice per group (D). ${ }^{\star} P<0.05,{ }^{* *} P<0.01$ versus PBS (i.p.), ${ }^{\dagger} P<0.05,{ }^{\dagger \dagger} P<0.01$. D, day.

effect of SP on Treg function was examined. Tregs isolated from normal mice were cultured in the presence of SP with or without Spantide I, an NK-1R antagonist. After 48 hours of culture, Tregs were analyzed for their inhibitory phenotypes and suppressive function. The data show that SP-treated Tregs expressed significantly lower levels of Foxp3 $(P=0.0101)$ and the inhibitory molecules CTLA-4 $(P \leq 0.001)$ and PD-1 $(P=0.039)$ compared with control. However, these phenotypic changes in Tregs were reversed with the addition of the NK-1R antagonist (Spantide I) to the culture. Tregs treated with both SP and Spantide I had higher expression levels of Foxp3 $(P=0.005)$, CTLA-4 $(P=0.0097)$, and PD-1 $(P=0.043)$ compared with SP-treated Tregs (Figure 3A). No significant changes were observed in the expression of GITR or lymphocyte activation gene-3 by Tregs after SP treatment (data not shown). The function of cultured Tregs was further evaluated by examining the secreted anti-inflammatory cytokines TGF- $\beta$ and IL-10 in the supernatants. Both TGF- $\beta$ $(P=0.026)$ and IL-10 $(P=0.0026)$ protein levels were significantly reduced in the presence of SP compared with controls. Blockade of SP signaling by Spantide I inhibited the SP-induced decrease in TGF- $\beta$ production by Tregs $(P=0.038)$ compared with the SP-treated group (Figure 3B).

Finally, the suppressive function of cultured Tregs was assessed. Tregs cultured in the presence of SP exhibited significantly decreased capability in suppressing effector T-cell proliferation compared with the Tregs cultured alone $(P=0.013)$, and the addition of Spantide I along with SP prevented the functional impairment of cultured Tregs $(P=0.0047)$ compared with the SP-treated group (Figure 3C). To ensure that Spantide I did not have a direct effect on Treg function, Tregs were cultured with Spantide I. The data show that Spantide I alone does not significantly affect Treg phenotypes or functions (data not shown).

\section{In Vivo Treatment with an NK-1R Antagonist Reduces Corneal Epitheliopathy in DED}

Next, it was investigated whether blockade of SP by Spantide I can reduce DED severity by modulating the underlying immune responses. Spantide I was administered to mice daily by intraperitoneal injection from one day before DED induction until day 14. PBS-injected mice were used as controls. CFS scores were used to evaluate corneal epitheliopathy. The data show significantly lower CFS scores in Spantide I-treated mice compared with the control mice on days $7(P=0.03), 10(P=0.015)$, and 14 $(P<0.001)$ (Figure 4A).

\section{NK-1R Antagonist Prevents Treg Dysfunction in DED}

To understand the underlying mechanisms by which Spantide I ameliorates DED in vivo, Treg frequencies, inhibitory phenotypes, and suppressive functions in treated DED mice were further analyzed. No significant differences were observed in the frequencies of Tregs as a proportion of total $\mathrm{CD}^{+}{ }^{+} \mathrm{T}$ cells in DLNs of normal (non-DED) mice, PBStreated DED mice, and Spantide I-treated DED mice (Figure 4B). However, consistent with a previous report on Treg dysfunction in DED, it was observed that Tregs derived from the PBS-treated group had significantly compromised suppressive function compared with normal 
A
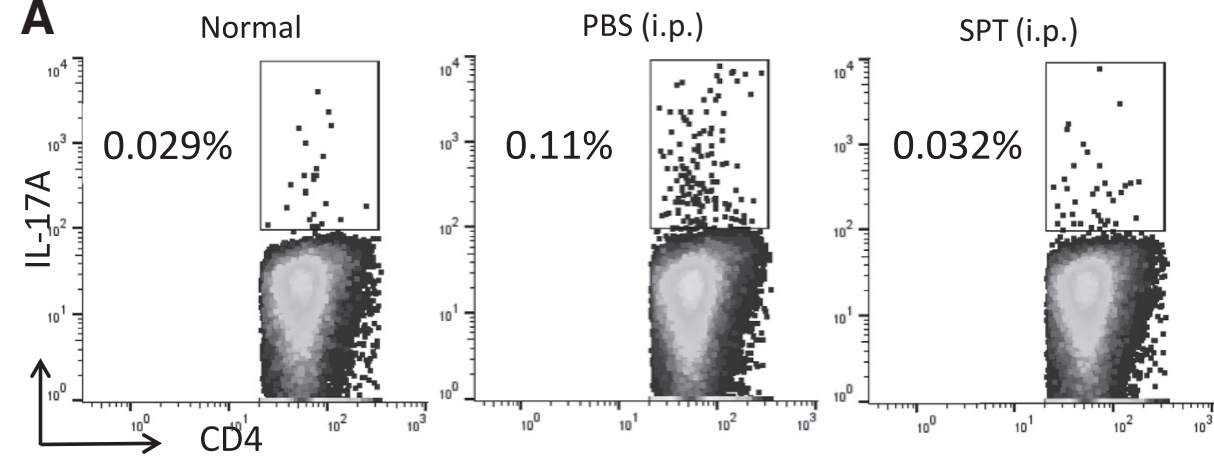

C
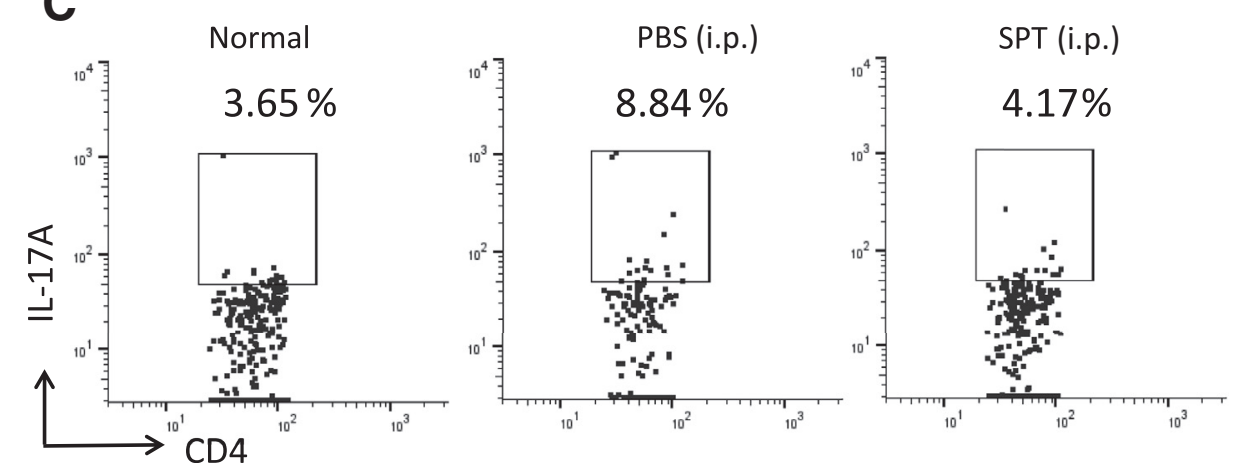

B

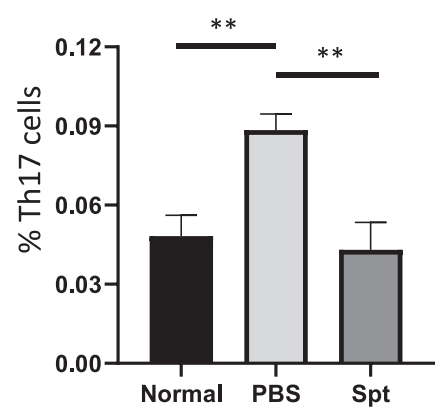

D

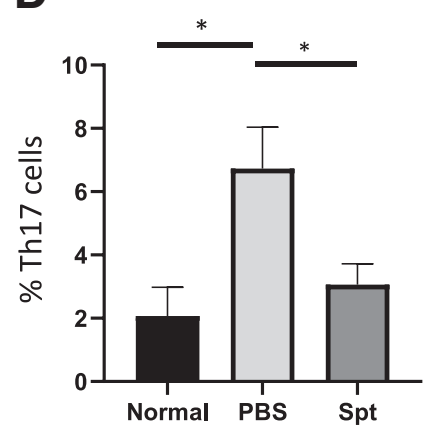

Figure 5 In vivo blockade of substance $\mathrm{P}$ (SP) signaling through systemic administration of Spantide I (SPT) suppresses T helper 17 (Th17) cell response. At day 14, frequencies of IL-17-producing $\mathrm{CD}^{+}{ }^{+}$T cells (Th17 cells) in draining lymph nodes (A and $\left.\mathbf{B}\right)$ and conjunctivae (C and $\left.\mathbf{D}\right)$ were analyzed by using flow cytometry. Phosphate-buffered saline (PBS) (i.p.) indicates intraperitoneal injection of PBS; SPT (i.p.), intraperitoneal injection of Spantide I (36 $\mu$ g per mouse daily). Representative flow cytometry dot plots are shown on the left (A and $\mathbf{C})$, and bar graph summaries are shown on the right (B and $\mathbf{D})$. Data are expressed as means \pm SEM of three independent experiments. $n=4$ draining lymph nodes and conjunctiva, with four tissues pooled together as one sample (B and D). ${ }^{*} P<0.05,{ }^{*} P P<0.01$.

mice. ${ }^{12}$ In addition, treatment of DED mice with Spantide I was efficacious in preserving the function of Tregs in suppressing effector T-cell proliferation (percent suppression, $50.0 \pm 3.2 \%$ versus $34.1 \pm 3.7 \% ; P=0.027$ ) (Figure $4 \mathrm{C}$ ). The protein levels of TGF- $\beta$ and IL-10 in DLNs were also evaluated. The levels of TGF- $\beta$ and IL-10 were significantly higher in Spantide I-treated DED mice $(P<0.001$ for both cytokines) compared with the PBS-treated group (Figure 4D).

\section{NK-1R Antagonist Suppresses Th17 Response in DED}

The frequencies of Th17 cells, the principal effector T-cell population implicated in the pathogenesis of DED, were analyzed. ${ }^{12}$ Systemic treatment with Spantide I significantly reduced Th17 frequencies in DLNs compared with the PBStreated group $(0.048 \pm 0.008 \%$ versus $0.088 \pm 0.005 \%$; $P=0.005$ ) (Figure 5, A and B). Similar results were observed in the conjunctiva, with the control-treated group having significantly higher Th17 cell frequencies $(6.7 \pm 1.1 \%)$ compared with the Spantide I-treated mice $(3.1 \pm 0.6 \% ; P=0.039)$ (Figure $5, \mathrm{C}$ and $\mathrm{D}$ ).

\section{Discussion}

It is known that the adoptive transfer of $\mathrm{CD}^{+} \mathrm{T}$ cells from DED to normal mice induces severe inflammation at the ocular surface, and Treg suppressive function in DED is impaired even though there is no significant change in their frequencies. ${ }^{12,36}$ These data suggest that the immune homeostasis breakdown occurs as a consequence of impaired Treg function, leading to unchecked effector $\mathrm{CD}^{+}{ }^{+} \mathrm{T}$-cell function in DED. The novelty of the current study lies in showing the increased expression of SP in the lymphoid tissues in DED, which directly impairs Treg suppressive function. Furthermore, blockade of SP signaling prevents SPinduced Treg dysfunction both in vitro and in vivo and significantly suppresses the severity of DED.

Changes in expression of SP in human tears have been reported in various conditions, such as allergic conjunctivitis and corneal hypoesthesia, and following laser-assisted in situ keratomileusis. ${ }^{37-39}$ Evidence suggests that SP plays a critical role in augmenting inflammatory responses principally by high-affinity binding to the NK-1R present on dendritic cells and macrophages. ${ }^{40}$ In addition, Marriott 
et $\mathrm{al}^{21}$ reported that SP and various other pro-inflammatory cytokines increase NK-1 $\mathrm{R}^{+}$macrophages. Blockade of NK$1 \mathrm{R}$ leads to decreases in the influx of neutrophils and $\mathrm{CD} 4^{+}$ $\mathrm{T}$ cells in herpetic stromal keratitis, suppressing the expression of interferon- $\gamma$, IL-18, tumor necrosis factor- $\alpha$, and IL- 6 in corneal infection, and enhancing IL-10. ${ }^{41-43}$ Recently, it was reported that topical blockade of NK-1R suppresses maturation and activation of corneal $\mathrm{CD} 11 \mathrm{~b}^{+}$ cells in DED. ${ }^{26}$ In aggregate, all of the aforementioned studies suggest an intimate link between SP and innate immune amplification.

SP is expressed by various cell types, regulating innate immune cell migration, activation of neutrophils, and promoting increases of IL- $1 \beta$, IL- 6 , tumor necrosis factor- $\alpha$, and IL-23 levels by monocytes. ${ }^{19,44,45}$ However, very little is known about the direct effect of SP on Treg phenotype and function. Herein, we investigated the phenotypic and functional changes of Tregs in DLNs in a validated murine model of DED and correlated these findings to SP expression levels and Treg functions. This study found that SP impairs Treg function by reducing the expression of Foxp3 and other inhibitory membrane-bound molecules such as CTLA-4 and PD-1, as well as secreted cytokines, TGF- $\beta$, and IL-10. In vitro, antagonizing NK-1R prevents SP-induced Treg dysfunction as well as recovered TGF- $\beta$ levels in the culture, although the SP-induced decrease of IL-10 secretion by Tregs is not prevented by blocking NK-1R. In the in vitro cultures, TGF- $\beta$ may play a dominant role for Treg functions, as evidenced by its 30 -fold higher levels compared with IL-10 in the supernatant. Interestingly, antagonizing NK$1 \mathrm{R}$ in vivo prevents Treg dysfunction by restoring both TGF$\beta$ and IL-10 expressions in DED mice, which is consistent with previous reports. ${ }^{42,43}$ A possible explanation for this differential effect of NK-1R antagonism on IL-10 expression between in vitro and in vivo may be a consequence of the effect of NK1R blockade on other immune cells producing IL-10, such as dendritic cells, $\mathrm{T}$ helper 1 and 2 cells, natural killer cells, and macrophages. ${ }^{46}$

SP is known to promote IL-17 secretion by human memoryphenotype T cells. ${ }^{45}$ Our results show that treatment with an NK-1R antagonist suppresses Th17 responses on the ocular surface, as well as in the lymphoid tissue in DED, and consequently reduces disease severity. This scenario suggests that SP/NK-1R antagonism may be a viable therapeutic strategy for suppressing DED severity by rescuing Treg dysfunction and suppressing the Th17 response, which plays an essential function in DED pathogenesis. ${ }^{12,47}$

\section{Conclusions}

The current findings provide new insights into the effects of SP on the phenotype and function of Tregs. We show for the first time that SP directly induces Treg dysfunction, and blockade of SP-NK-1R signaling effectively abolishes such an effect. Using the DED model, the benefits of NK-1R blockade in improving Treg functions and reducing corneal epitheliopathy are further validated, indicating a broad translational potential of blocking SP-NK-1R signaling to treat autoimmune diseases.

\section{Acknowledgment}

We thank Santanu Mukherjee, Ph.D., for his scientific feedback and editing the manuscript.

\section{Author Contributions}

Y.T., S.K.C., Y.C., and R.D. designed the study; Y.T., A.M., T.H.D., and Y.C. acquired data; Y.T. and Y.C. analyzed the data; Y.T. prepared the manuscript; A.A., R.B.S., Y.C., and R.D. revised the manuscript; and S.K.C., Y.C., and R.D. supervised the study; all authors read and approved the final manuscript.

\section{References}

1. The definition and classification of dry eye disease: report of the Definition and Classification Subcommittee of the International Dry Eye WorkShop. Ocul Surf 2007, 5:75-92

2. Shikari H, Antin JH, Dana R: Ocular graft-versus-host disease: a review. Surv Ophthalmol 2013, 58:233-251

3. Markey KA, MacDonald KP, Hill GR: The biology of graft-versushost disease: experimental systems instructing clinical practice. Blood 2014, 124:354-362

4. Brito-Zerón P, Baldini C, Bootsma H, Bowman SJ, Jonsson R, Mariette X, Sivils K, Theander E, Tzioufas A, Ramos-Casals M: Sjögren syndrome. Nat Rev Dis Primers 2016, 2:16047

5. Smolen JS, Aletaha D, McInnes IB: Rheumatoid arthritis [Erratum appeared in in Lancet 2016, 388:1984]. Lancet 2016, 388:2023-2038

6. The epidemiology of dry eye disease: report of the Epidemiology Subcommittee of the International Dry Eye WorkShop (2007). Ocul Surf 2017, 5:93-107

7. Yu J, Asche CV, Fairchild CJ: The economic burden of dry eye disease in the United States: a decision tree analysis. Cornea 2011, 30:379-387

8. Stevenson W, Chauhan SK, Dana R: Dry eye disease: an immunemediated ocular surface disorder. Arch Ophthalmol 2012, 130: $90-100$

9. Foulsham W, Marmalidou A, Amouzegar A, Coco G, Chen Y, Dana R: Review: the function of regulatory $\mathrm{T}$ cells at the ocular surface. Ocul Surf 2017, 15:652-659

10. Chen Y, Chauhan SK, Lee HS, Saban DR, Dana R: Chronic dry eye disease is principally mediated by effector memory Th17 cells. Mucosal Immunol 2014, 7:38-45

11. Barabino S, Chen Y, Chauhan S, Dana R: Ocular surface immunity: homeostatic mechanisms and their disruption in dry eye disease. Prog Retin Eye Res 2012, 31:271-285

12. Chauhan SK, El Annan J, Ecoiffier T, Goyal S, Zhang Q, Saban DR, Dana R: Autoimmunity in dry eye is due to resistance of Th17 to Treg suppression. J Immunol 2009, 182:1247-1252

13. Chang MM, Leeman SE, Niall HD: Amino-acid sequence of substance P. Nat New Biol 1971, 232:86-87

14. De Felipe C, Herrero JF, O'Brien JA, Palmer JA, Doyle CA Smith AJ, Laird JM, Belmonte C, Cervero F, Hunt SP: Altered nociception, analgesia and aggression in mice lacking the receptor for substance P. Nature 1998, 392:394-397

15. Suvas $\mathrm{S}$ : Role of substance $P$ neuropeptide in inflammation, wound healing, and tissue homeostasis. J Immunol 2017, 199:1543-1552 
16. Maggi CA: Tachykinins and calcitonin gene-related peptide (CGRP) as co-transmitters released from peripheral endings of sensory nerves. Prog Neurobiol 1995, 45:1-98

17. Holzer P: Local effector functions of capsaicin-sensitive sensory nerve endings: involvement of tachykinins, calcitonin gene-related peptide and other neuropeptides. Neuroscience 1988, 24:739-768

18. Mantyh PW: Neurobiology of substance P and the NK1 receptor. J Clin Psychiatry 2002, 63 Suppl 11:6-10

19. Mashaghi A, Marmalidou A, Tehrani M, Grace PM, Pothoulakis C, Dana R: Neuropeptide substance $\mathrm{P}$ and the immune response. Cell Mol Life Sci 2016, 73:4249-4264

20. Watanabe M, Nakayasu K, Iwatsu M, Kanai A: Endogenous substance P in corneal epithelial cells and keratocytes. Jpn J Ophthalmol 2002, 46:616-620

21. Marriott I, Bost KL: IL-4 and IFN-gamma up-regulate substance P receptor expression in murine peritoneal macrophages. J Immunol 2000, 165:182-191

22. Ho WZ, Lai JP, Zhu XH, Uvaydova M, Douglas SD: Human monocytes and macrophages express substance $\mathrm{P}$ and neurokinin-1 receptor. J Immunol 1997, 159:5654-5660

23. Lambrecht BN, Germonpré PR, Everaert EG, Carro-Muino I, De Veerman M, de Felipe C, Hunt SP, Thielemans K, Joos GF, Pauwels RA: Endogenously produced substance $\mathrm{P}$ contributes to lymphocyte proliferation induced by dendritic cells and direct TCR ligation. Eur J Immunol 1999, 29:3815-3825

24. Bignami F, Rama P, Ferrari G: Substance P and its inhibition in ocular inflammation. Curr Drug Targets 2016, 17:1265-1274

25. O'Connor TM, O'Connell JO, O'Brien DI, Goode T, Bredin CP, Shanahan F: The role of substance P in inflammatory disease. J Cell Physiol 2004, 201:167-180

26. Lisowska B, Lisowski A, Siewruk K: Substance P and chronic pain in patients with chronic inflammation of connective tissue. PLoS One 2015, 10:e0139206

27. Keeble JE, Brain SD: A role for substance P in arthritis? Neurosci Lett 2004, 361:176-179

28. Weinstock JV: Substance $P$ and the regulation of inflammation in infections and inflammatory bowel disease. Acta Physiol (Oxf) 2015, $213: 453-461$

29. Yu M, Lee SM, Lee H, Amouzegar A, Nakao T, Chen Y, Dana R: Neurokinin-1 receptor antagonism ameliorates dry eye disease by inhibiting antigen-presenting cell maturation and $\mathrm{T}$ helper 17 cell activation. Am J Pathol 2020, 190:125-133

30. The Association for Research in Vision and Opthamology, ARVO Statement for the Use of Animals in Ophthalmic and Vision Research. Rockville, MD: The Association for Research in Vision and Opthamology, 1995

31. Chen Y, Chauhan SK, Tan X, Dana R: Interleukin-7 and -15 maintain pathogenic memory Th17 cells in autoimmunity. J Autoimmun 2017, $77: 96-103$

32. Lemp MA: Report of the National Eye Institute/Industry workshop on clinical trials in dry eyes. CLAO J 1995, 21:221-232

33. Chen Y, Chauhan SK, Lee HS, Stevenson W, Schaumburg CS, Sadrai Z, Saban DR, Kodati S, Stern ME, Dana R: Effect of desiccating environmental stress versus systemic muscarinic AChR blockade on dry eye immunopathogenesis. Invest Ophthalmol Vis Sci 2013, 54:2457-2464

34. Hori S, Nomura T, Sakaguchi S: Control of regulatory $\mathrm{T}$ cell development by the transcription factor Foxp3. Science 2003, 299: 1057-1061

35. Fontenot JD, Gavin MA, Rudensky AY, Pillars Article: Foxp3 programs the development and function of $\mathrm{CD} 4+\mathrm{CD} 25+$ regulatory $\mathrm{T}$ cells. Nat. Immunol. 2003. 4: 330-336. J Immunol 2017, 198: 986-992

36. Niederkorn JY, Stern ME, Pflugfelder SC, De Paiva CS, Corrales RM, Gao J, Siemasko K: Desiccating stress induces T cellmediated Sjögren's syndrome-like lacrimal keratoconjunctivitis. J Immunol 2006, 176:3950-3957

37. Fujishima H, Takeyama M, Takeuchi T, Saito I, Tsubota K: Elevated levels of substance $\mathrm{P}$ in tears of patients with allergic conjunctivitis and vernal keratoconjunctivitis. Clin Exp Allergy 1997, 27:372-378

38. Yamada M, Ogata M, Kawai M, Mashima Y: Decreased substance P concentrations in tears from patients with corneal hypesthesia. Am J Ophthalmol 2000, 129:671-672

39. Chao C, Golebiowski B, Zhao X, Chen S, Zhou S, Stapleton F: Long-term effects of LASIK on corneal innervation and tear neuropeptides and the associations with dry eye. J Refract Surg 2016 , $32: 518-524$

40. Marriott I, Bost KL: Expression of authentic substance P receptors in murine and human dendritic cells. J Neuroimmunol 2001, 114 $131-141$

41. Twardy BS, Channappanavar R, Suvas S: Substance P in the corneal stroma regulates the severity of herpetic stromal keratitis lesions. Invest Ophthalmol Vis Sci 2011, 52:8604-8613

42. Lighvani S, Huang X, Trivedi PP, Swanborg RH, Hazlett LD: Substance $\mathrm{P}$ regulates natural killer cell interferon-gamma production and resistance to Pseudomonas aeruginosa infection. Eur J Immunol 2005, 35:1567-1575

43. Hazlett LD, McClellan SA, Barrett RP, Liu J, Zhang Y, Lighvani S: Spantide I decreases type I cytokines, enhances IL-10, and reduces corneal perforation in susceptible mice after Pseudomonas aeruginosa infection. Invest Ophthalmol Vis Sci 2007, 48:797-807

44. Serra MC, Bazzoni F, Della Bianca V, Greskowiak M, Rossi F: Activation of human neutrophils by substance P. Effect on oxidative metabolism, exocytosis, cytosolic $\mathrm{Ca} 2+$ concentration and inositol phosphate formation. J Immunol 1988, 141:2118-2124

45. Cunin P, Caillon A, Corvaisier M, Garo E, Scotet M, Blanchard S, Delneste Y, Jeannin P: The tachykinins substance P and hemokinin-1 favor the generation of human memory Th17 cells by inducing IL-1 [beta], IL-23, and TNF-like 1A expression by monocytes. J Immunol 2011, 186:4175-4182

46. Moore KW, de Waal Malefyt R, Coffman RL, O'Garra A: Interleukin-10 and the interleukin-10 receptor. Annu Rev Immunol 2001 19:683-765

47. De Paiva CS, Chotikavanich S, Pangelinan SB, Pitcher JD III, Fang B, Zheng X, Ma P, Farley WJ, Siemasko KF, Niederkorn JY, Stern ME, Li DQ, Pflugfelder SC: IL-17 disrupts corneal barrier following desiccating stress. Mucosal Immunol 2009, 2:243-253 